\title{
Upflowing Ionospheric Ions and Electrons in the Cusp-Cleft Region
}

\author{
B. THELIN and R. LUNDIN \\ Swedish Institute of Space Physics, P.O. Box 812, S-981 28 Kiruna, Sweden
}

(Received January 25, 1990; Accepted March 5, 1990)

\begin{abstract}
Measurements of electron and ion beams and ion conics from the polar orbiting Viking satellite have been obtained in the cusp-cleft region. Frequencies of occurrence and relative frequencies of occurrence have been calculated in this region for studies of the distributions in MLT, Inv. Lat. and altitude during the period of study (March-June 1986). It was observed that the electron beams were more frequent in the dawn sector than in the dusk sector. These observations were similar and correlated to the ion conics and are compared in this study. On the other hand, an anticorrelation between electron- and ion-beams was also observed. The altitude dependence of the ion conics and electron beams showed a steep increase in frequency above $10000 \mathrm{~km}$ up to the satellite apogee at about $13500 \mathrm{~km}$.
\end{abstract}

\section{Introduction}

The existence of acceleration processes of ions and electrons along magnetic field lines by quasi-static parallel electric fields is by now an established fact. The results from the S3-3 satellite clearly demonstrate this (SHELLEY et al., 1976) but the results also showed that ions may become accelerated perpendicular to the magnetic field, producing ion conics.

The ion conics are often elevated, where the lowest energy particles are field aligned indicating a combination of perpendicular and parallel acceleration of the ions. Several authors have reported observations of ion conics together with electron beams (HULTQVIST et al., 1988). A frequent observation in many of the conic generation regions is the occurrence of upward electron beams. According to a paper by DUSENBERY and LYONS (1981) ion conics are related to downward currents. However, the ion conics also exist in regions with bidirectional streaming electrons or in regions with no significant net upward electron flow.

With the Viking satellite the ion conics were observed to be generated at practically all altitudes where Viking has been operated. Ion conics are also frequently associated with upward streaming, or bidirectionally streaming electrons, in agreement with the S3-3 and DE-1 observations (SHARP et al., 1980). The most likely process associated with the generation of ion conics is perpendicular heating of ions in the upper ionosphere. This heating process may result from wave particle interactions (KINTNER et al., 1979) or it may simply be due to strong perpendicular fluctuations of the electric fields recently shown by LUNDIN and HULTQVIST (1989).

Viking observations over the dayside oval have demonstrated that the dayside cuspcleft is an important source of plasma for the magnetosphere also during magnetically quiet periods. Newer analyses of the Viking data show that the prenoon sector of the cleft 
is dominated by ion conics and strong upward or bidirectionally streaming electron beams. The postnoon sector, on the other hand, is dominated by strong upward ion beams and downwards moving electrons, which is consistent with the current models by IIJIMA and POTEMRA (1978).

In this paper a statistical study has been performed in the cusp-cleft region concerning the distributions between upgoing ion conic events and upgoing electron beams using relative frequencies of occurrence. These studies have been performed for different MLT, Inv. Lat. and altitude locations, showing strong correlations.

\section{Instrumentation and Data Analysis Procedure}

The Viking satellite was placed in an eccentric orbit (apogee $13500 \mathrm{~km}$ and perigee $800 \mathrm{~km}$ ) and became operational in February 1986. The particle instruments (V3) on Viking consist of 7 spectrometer units and are described in detail by SANDAHL et al. (1985). The data used in this paper were recorded by the electron spectrometers (ESP 1) designed for measuring electrons in the energy range $(0.01-40) \mathrm{keV}$. The positive ions, on the other hand, were detected by the positive ion spectrometers (PISP 1/2) capable of measuring ions in the energy ranges (1.2-40) $\mathrm{keV}$ and $(0.04-1.2) \mathrm{keV}$. To give a broad statistical coverage in MLT, Inv. Lat., and altitude the data in this study were selected from the period March to June 1986. The analysis of the data was carried out by using visual examination of the energy versus time particle spectrograms.

These were divided into bins corresponding to a volume of $1000 \mathrm{~km}$ in altitude, 1 degree in invariant latitude, and 1 hour in magnetic local time.

The upward flowing electron beams have a peak flux along the magnetic field lines in a pitch angle interval of $180 \pm 5^{\circ}$. The mean value of each beam was plotted versus magnetic local time, invariant latitude and altitude and the electron beams in this investigation were all interpreted as "clear cases" and have energies in the range (0.01-40) $\mathrm{keV}$. The ion conics, on the other hand, were spanning a pitch angle interval of $180 \pm 90^{\circ}$. In statistical investigations one usually uses the frequency of occurrence (YAU et al., 1984), defined as:

$$
f(\mathrm{MLT}, \mathrm{IL}, \mathrm{A})=n(\mathrm{MLT}, \mathrm{IL}, \mathrm{A}) / N(\mathrm{MLT}, \mathrm{IL}, \mathrm{A}),
$$

where $n$ (MLT, IL, A) is the number of events in the bin labeled (MLT, IL, A) and $N(\mathrm{MLT}, \mathrm{IL}, \mathrm{A})$ is the number of samples in a certain bin, which means the number of satellite orbits. $f(\mathrm{MLT}, \mathrm{IL}, \mathrm{A})$ is evidently the frequency of occurrence of an electron beam within the volume (MLT, IL, A).

In this paper relative frequency of occurrence defined as

$$
f(\mathrm{rel})=\frac{n(\mathrm{MLT}, \mathrm{IL}, \mathrm{A}) N(\max )}{\sum n(\mathrm{MLT}, \mathrm{IL}, \mathrm{A}) N(\mathrm{MLT}, \mathrm{IL}, \mathrm{A})}
$$

was used. $n$ (MLT, IL, A) is here the number of events in the bin labeled (MLT, IL, A), and $\sum n$ (MLT, IL, A) is the sum of the number of events studied in all bins. $N(\max ) / N(\mathrm{MLT}, \mathrm{IL}, \mathrm{A})$ is a normalisation factor of the satellite orbits, where $N(\max )$ is the number of satellite orbits at apogee. As the factor $N(\max ) / \Sigma n(\mathrm{MLT}, \mathrm{IL}, \mathrm{A})$ is a constant and $n(\mathrm{MLT}, \mathrm{IL}, \mathrm{A}) / N(\mathrm{MLT}, \mathrm{IL}, \mathrm{A})$ is the same as Eq. (1), the relative 
frequency of occurrence is about the same as the usual frequency of occurrence except for the scale factor $N(\max ) / \sum n(\mathrm{MLT}, \mathrm{IL}, \mathrm{A})$. This scale factor is, of course, different between electrons and ions. It has shown to be an advantage to use relative frequencies sometimes, especially in studies as those of this paper, where altitude profiles at different conditions are studied. Obviously, it is an advantage to normalise with $\Sigma n$ (MLT, IL, A) when comparing frequencies from different processes versus each other.

\section{Results and Discussion}

Figures 1 and 2 show the frequency of occurrence $(f)$ and the relative frequency of occurrence $(f(\mathrm{rel}))$ of upflowing ion conics and beams when they are grouped into one hour magnetic local time intervals and integrated over all invariant latitudes and altitudes at positive and negative $B_{y}$ components. As there are more cases for the $B_{y}>0$ alternative compared to the $B_{y}<0$ alternative in Fig. 1, the frequencies of occurrence are about twice as high when $B_{y}>0$ compared to when $B_{y}<0$. On the other hand, if relative frequencies of occurrence are used, about equal relative frequencies between the positive and negative $B_{y}$ components are obtained. Similar graphs as those of Fig. 1 are also shown in Fig. 2 for the ion beams. It is obvious from these plots that the beams dominate at dusk while the conics dominate at dawn with relative frequencies of about 0.3. The same thing is observed for both positive and negative $B_{y}$ components with a relative frequency of about 0.3 . In Figs. 1 and 2 a deep minimum at noon is also observed. A steeper increase of the curve from that minimum to the dusk maximum was also observed when $B_{y}<0$ compared to $B_{y}>0$. This means that the asymmetry effect mentioned above is also observed separately for upflowing beams and conical distributions.

One probable conclusion from this is that the ion conics are observed on the whole dayside and will be transformed into ion beams on the afternoon side due to the electric convection field. It is also logical to presume that at higher altitudes an ion conic will have an appearance that looks like an ion beam for a nonideal instrument. Therefore, it has become still more important to use only "clear cases" in this statistical investigation. Figure 3 shows the MLT distribution of the electron beams. It is clearly seen from this picture that the abundances of electron beams seem to be higher in the dawn sector than in the dusk sector. There is also a sort of "hole" in the abundances of the electron beams observed near noon. The location of this "hole" is consistent with the cusp MLT location and has earlier been observed in a paper by THELIN et al. (1989), where the location of upflowing ions was studied with the Viking satellite. In that paper a similar "hole" in the abundances of the ion conic- and beam-events was discovered around noon. The ion conics were more frequent in the dawn sector, which is similar to the electron beam observations; the ion beams, on the other hand, were more frequent in the dusk sector.

The altitude dependence of ion beams and conics was also investigated and is shown in Fig. 4. The frequency of occurrence and the relative frequency of occurrence for the ion beams studied increase with altitude from $5000 \mathrm{~km}$ to $10000 \mathrm{~km}$. For greater altitudes they stay fairly constant up to the satellite apogee. On the other hand, the occurrence frequency of the ion conics increases steadily from $7000 \mathrm{~km}$ to the satellite apogee $(13500$ $\mathrm{km}$ ). From $12000 \mathrm{~km}$ upwards the frequency of occurrence and the relative frequency of occurrence are about 2-3 times as high for the ion conics as for the ion beams. The results of Fig. 4 agree well with the results from the S3-3 and the DE satellites. Another way of illustrating the correlation between upgoing electron beams and ion conics is to plot the 

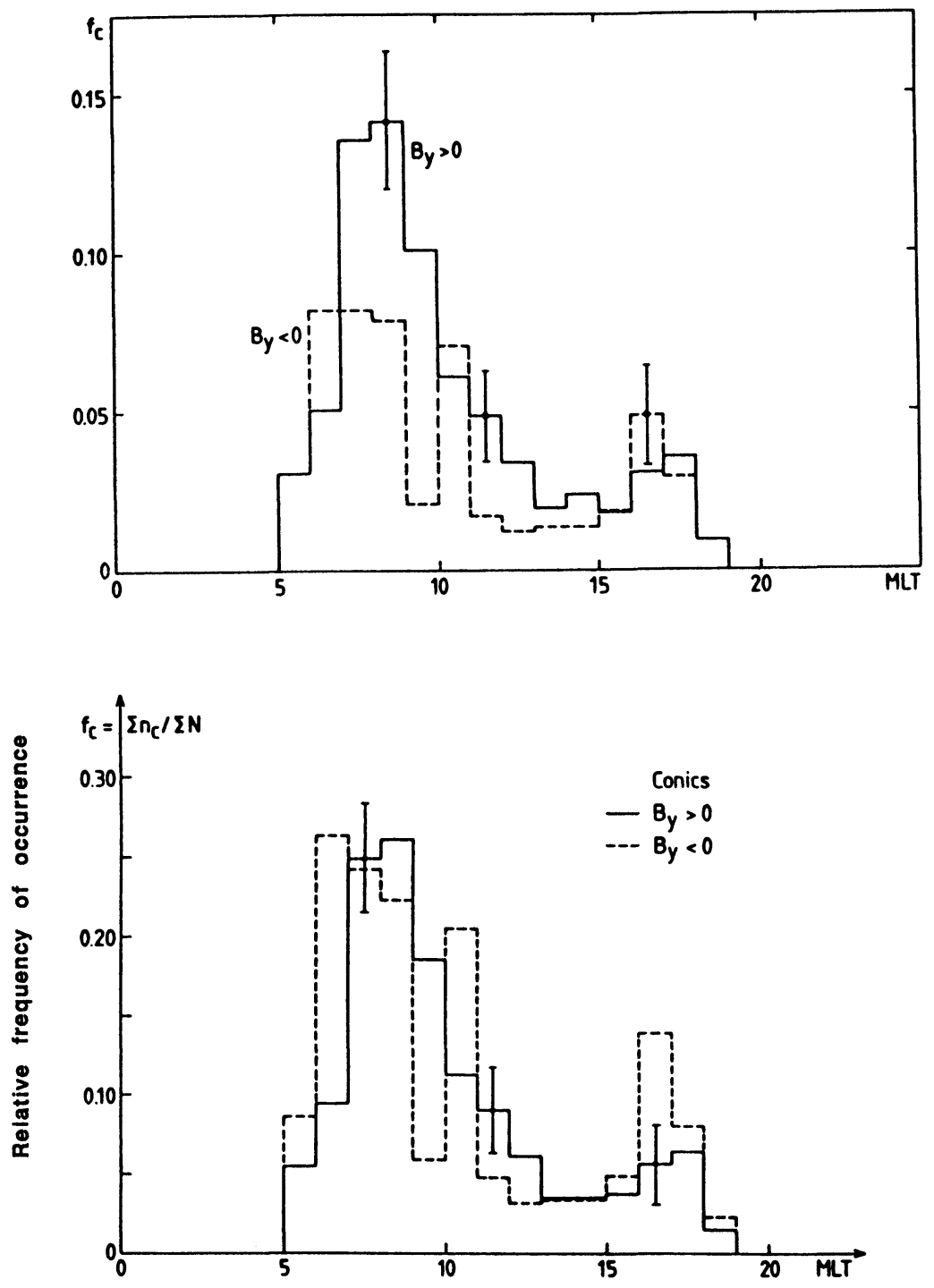

Fig. 1. Frequency of occurrence and relative frequency of occurrence of upflowing ion conics events versus magnetic local time (MLT) for positive and negative IMF $B_{y}$ components.

relative frequencies of occurrence versus each other for each bin in MLT, Inv. Lat., and altitude studied. These results are illustrated in Figs. 5(a), (b), and (c), which gave correlation coefficients of $0.96,0.97$, and 0.98 for the correlations in MLT, Inv. Lat., and altitude respectively. The points in these plots were also organised close to the 1-1 line, which reveals a nice correlation between upgoing electron beams and upgoing ion conic events. On the other hand, there was no correlation at all found between upgoing electron beams and upgoing ion beams. 

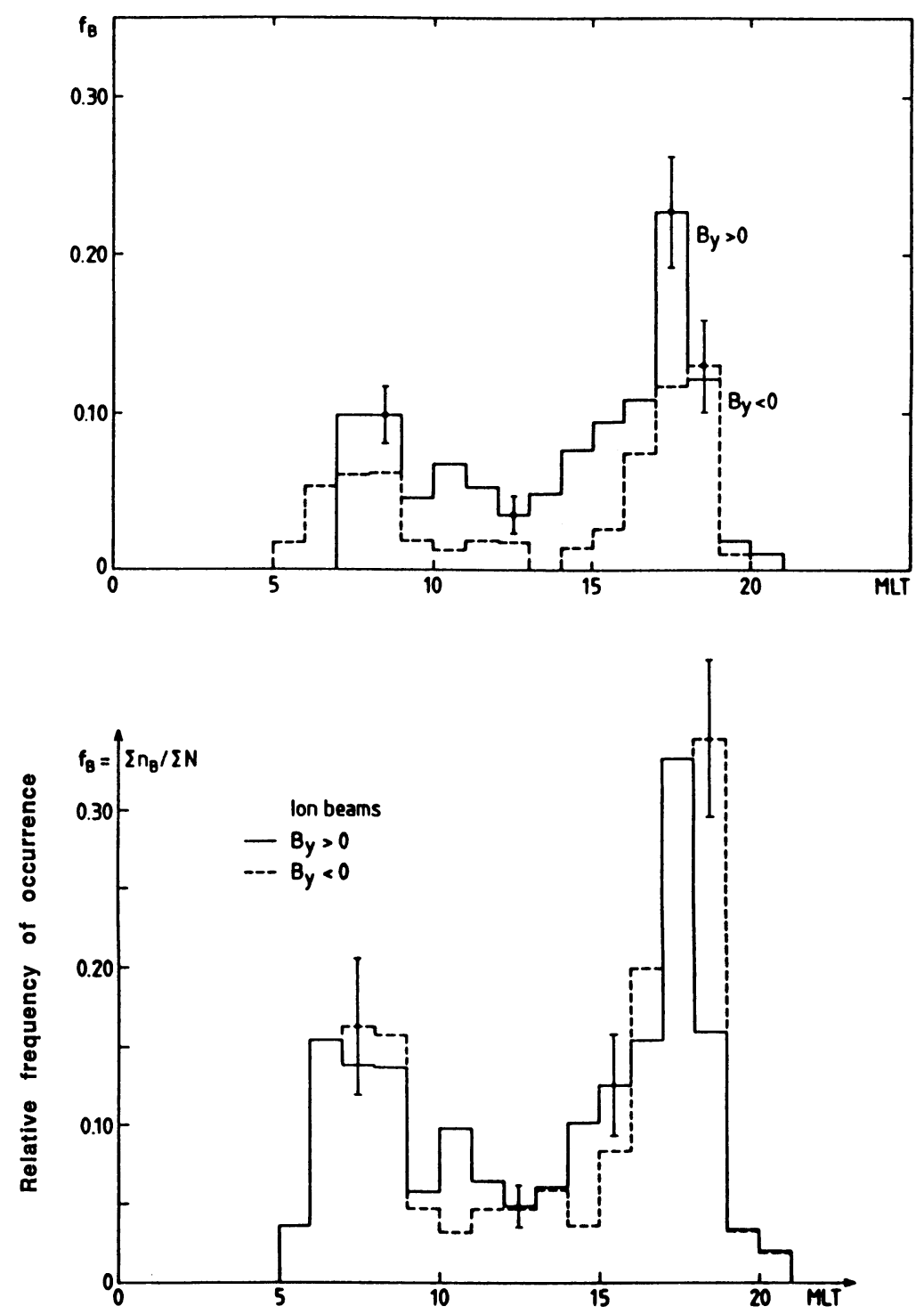

Fig. 2. Frequency of occurrence and relative frequency of occurrence of upflowing ion beams versus magnetic local time (MLT) for positive and negative IMF $B_{y}$ components.

The ion conics have an altitude profile which is very similar to the one of the upgoing electron beams, which can be seen ion Fig. 4. The relative frequencies at the disturbed situations seem to be slightly higher and a little more structured. Simultaneous observations of upward electron beams and ion conics have been discussed by several authors (SHARP et al., 1980). None of these authors indicate simultaneously occurring upward moving ions and electrons of similar energies or any influence of electric fields, because there was no electric field or wave data available. 


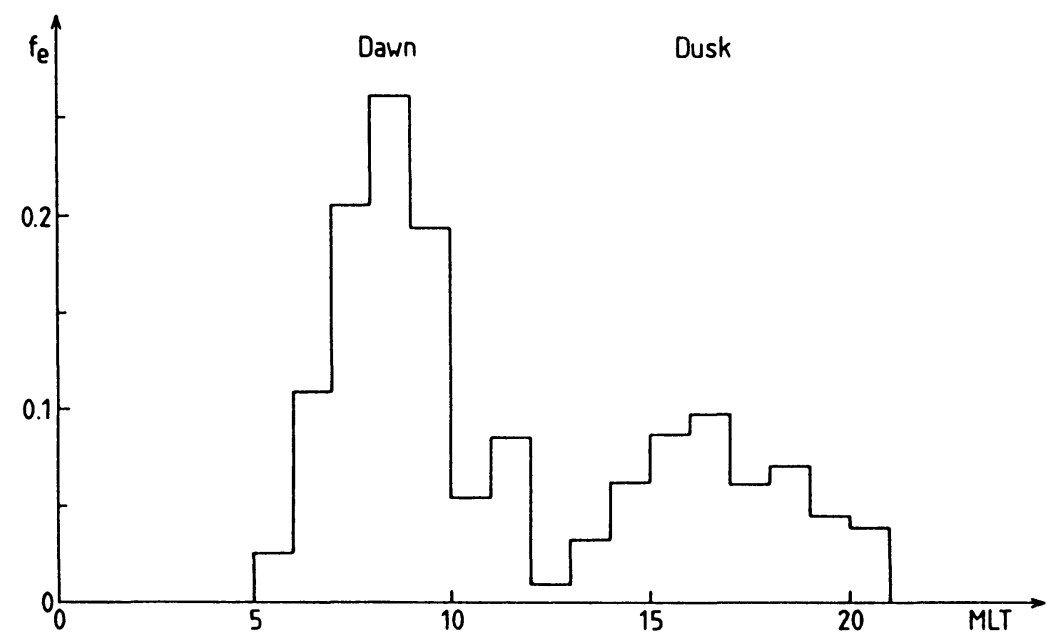

Fig. 3. Relative frequency of occurrence of upflowing electron beams versus magnetic local time (MLT).

GORNEY et al. (1981) have also observed ion conics together with upward fieldaligned electrons. They discussed the influence of downward electric field on the acceleration of ions and electrons and some trapping processes.

None of these authors have considered situations with coincident elevated ion conics and upward field-aligned electron beams, where they assume unrelated acceleration mechanisms, which occur at different altitudes. Observations by HULTQVIST et al. (1988) indicate that the two phenomena occur together and in the presence of very strong low-frequency fluctuations in the electric field. In a model by HULTQVIST (1988) it is suggested that both upward moving field-aligned electrons and ions are accelerated in the same direction and indicate that the two phenomena occur together and only in the presence of very strong low-frequency fluctuations in the electric field.

\section{Conclusion}

This paper has shown an observed correlation between ion conics and electron beams by plotting the relative frequencies of ion conics and electron beams at different MLT, Inv. Lat. and altitude distributions. This means that this correlation has shown to be invariant and it probably means that ion conics and electron beams emanate from the same physical process. On the other hand, an anticorrelation is found when comparing the occurrences of electron beams and ion beams. It is also observed that most electron beams and ion conics detected are more frequent in the dawn sector than in the dusk sector.

It is also found that the relative frequencies of occurrence for electron- and ionbeams and ion conics increase at disturbed situations compared with the undisturbed ones. The electron beams and ion conics increase at higher altitudes while the ion beams increase between $5000-10000 \mathrm{~km}$ altitude and reach a plateau at about $10000 \mathrm{~km}$. At 

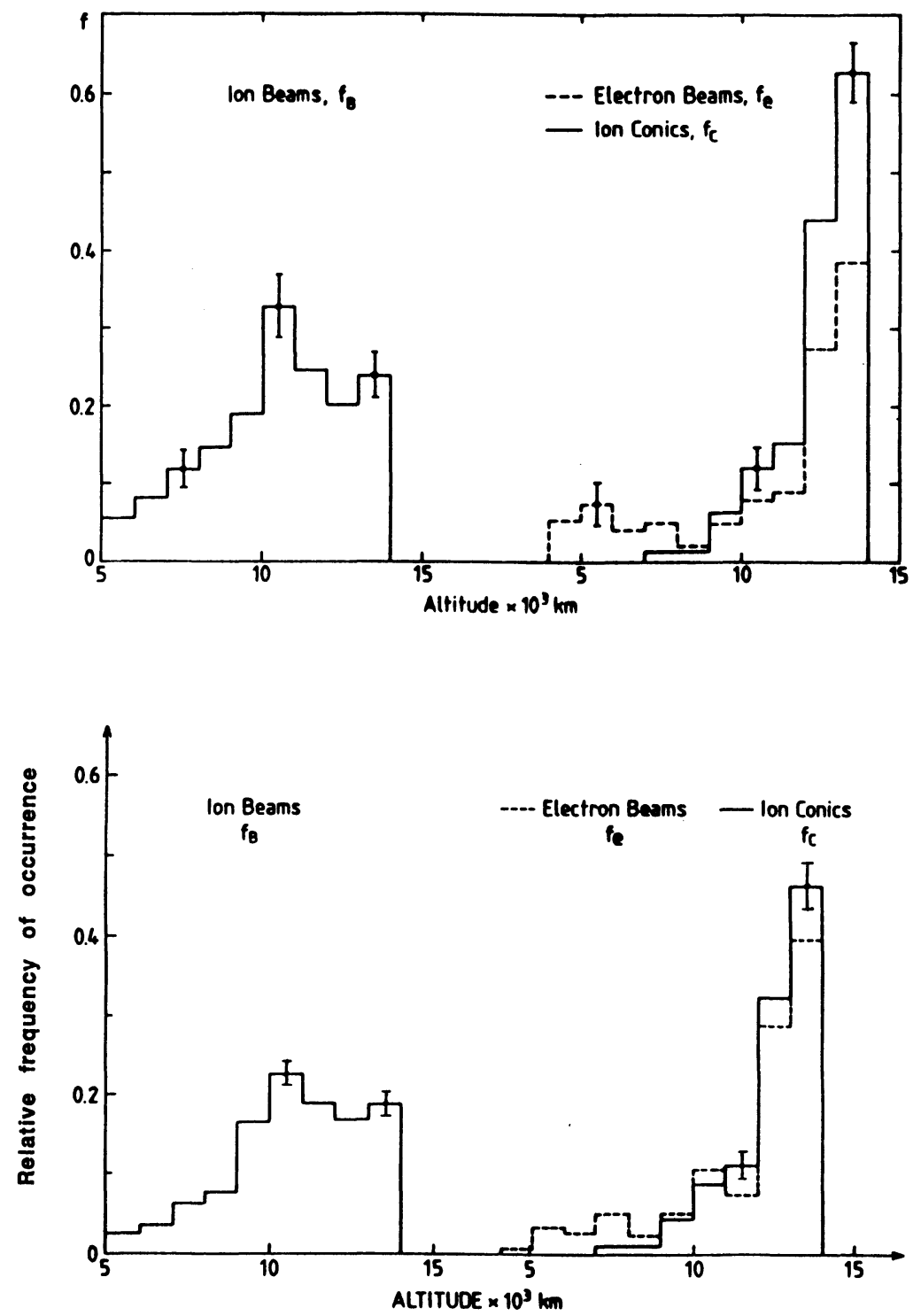

Fig. 4. Frequency of occurrence and relative frequency of occurrence of upflowing ion beams- and conics and electron beams versus altitude.

disturbed situations the upgoing electron beams are mostly observed above $11000 \mathrm{~km}$ while at undisturbed situations upgoing electron beams are observed from an altitude of $4000 \mathrm{~km}$. The distribution profile for electron- and ion-beams is also observed to be more structured at disturbed situations compared to the undisturbed ones. 


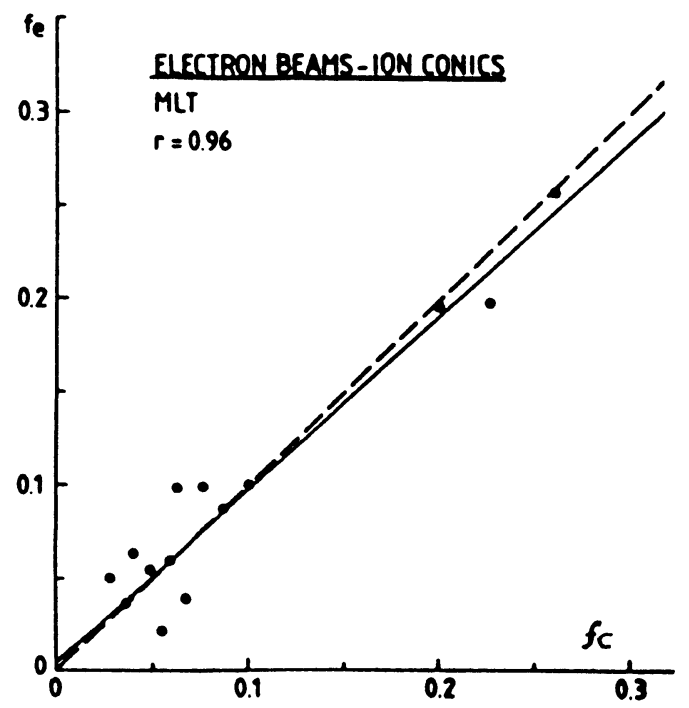

(a)

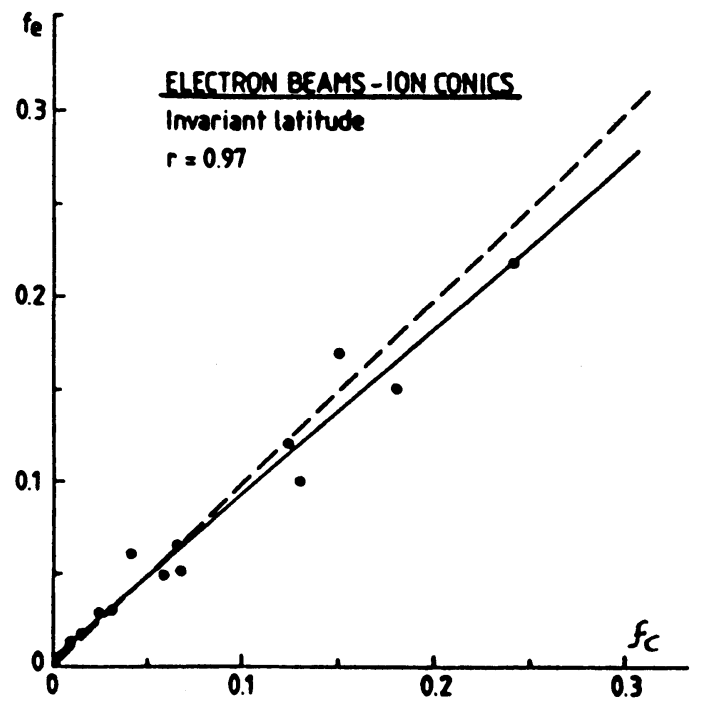

(b)

Fig. 5. Correlation studies between upgoing electron beams and ion conics versus MLT (a), Inv. Lat. (b), and altitude (c). 


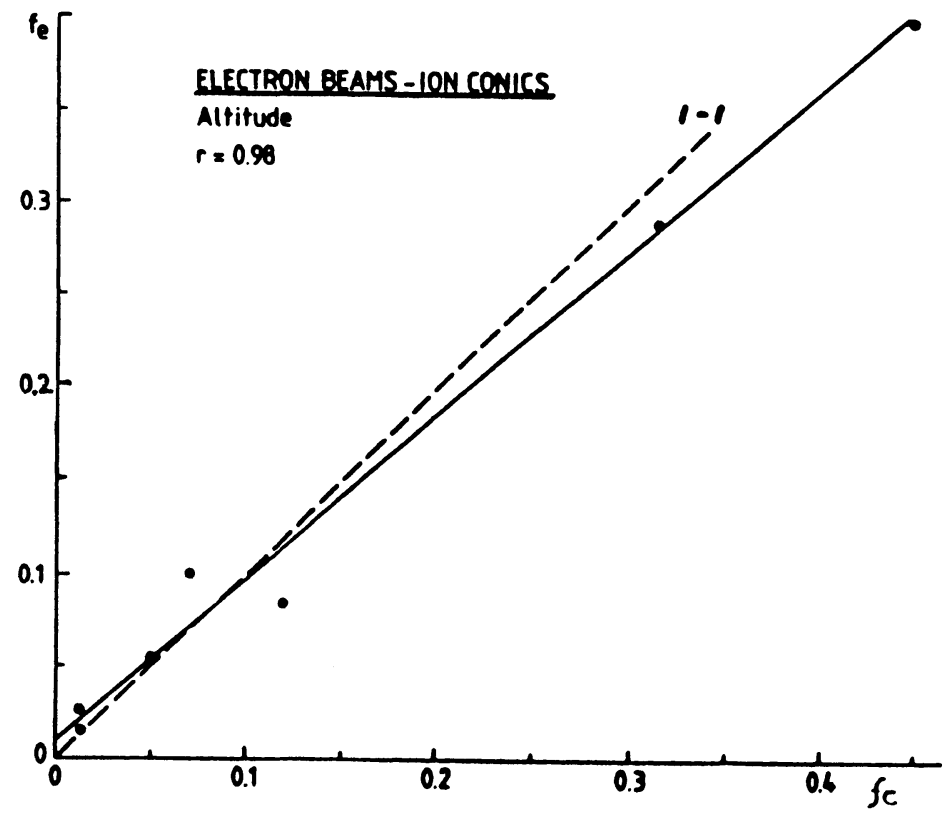

(c)

Fig. 5. (continued).

\section{REFERENCES}

DusEnBERy, P. B. and L. R. LyONS, Generation of ion conic distribution by upgoing ionospheric electrons, $J$. Geophys. Res., 86, 7627-7638, 1981.

Gorney, D. J., A. Clarke, D. Croley, J. F. Fennell, i. Luhmann, and P. F. Mizera, The distribution of ion beams and conics below $8000 \mathrm{~km}, J$. Geophys. Res., 86, 83, 1981.

HultQvist, B., On the acceleration of electrons and positive ions in the same direction along magnetic field lines, J. Geophys. Res., 93, 9777, 1988.

HultQvist, B., et al., Simultaneous observations of upward moving field aligned energetic electrons and ions on the auroral zone field lines, J. Geophys. Res., 93, 9765, 1988.

Iijima, T. and T. A. Potemra, Large scale characteristics of field aligned currents associated with substorms, $J$. Geophys. Res., 83, 559-615, 1978.

Kintner, P. M., M. C. Kelley, R. D. Sharp, A. G. Ghielmetti, M. Temerin, C. Cattell, P. F. Mizera, and J. F. FENNELL, Simultaneous observations of energetic $(\mathrm{keV})$ upstreaming ions and electrostatic hydrogen cyclotron waves, J. Geophys. Res., 84, 7201-7212, 1979.

Lundin, R. and B. HultQvist, Ionospheric plasma escape by high-altitude electric fields: Magnetic moment “pumping”, J. Geophys. Res., 94, 6665, 1989.

SAndahl, I., L. Eliasson, and R. Lundin, The hot plasma spectrometers on Viking, KGI preprint 077 , Feb. 1985.

Sharp, R. D., E. G. Shelley, R. G. Johnson, and A. G. Ghielmetti, Counterstreaming electron beams at altitudes of 1 Re over the auroral zone, J. Geophys. Res., 85, 92-100, 1980.

Shelley, E. G., R. D. Sharp; and R. G. Johnson, Satellite observations of an ionospheric acceleration mechanism, Geophys. Res. Lett., 3, 654-657, 1976.

Thelin, B., B. Aparicio, and R. Lundin, Observations of upflowing ionospheric ions in the mid altitude cusp-cleft region with the Viking satellite, submitted to J. Geophys. Res., 1989.

Yau, A. W., B. A. Whalen, W. K. Peterson, and E. G. Shelley, Distribution of upflowing ionospheric ions in the high-altitude polar cap and auroral ionosphere, J. Geophys. Res., 89, 5507-5522, 1984. 